\title{
Effect of Electric Field on Dielectric Constant and Loss Tangent in ADP Crystal
}

\author{
DEEPAK JOSHI* ${ }^{*}$ and TRILOK CHANDRA UPADHYAY
}

Physics Department, H.N.B. Garhwal University (A Central University), Srinagar (Garhwal) Uttarakhand- 246174, India

deepak_deepakjoshi@rediffmail.com

Received 5 October 2016 / Accepted 10 October 2016

\begin{abstract}
A four sublattice pseudospin lattice coupled mode model modified with third and fourthorder phonon anharmonic interaction terms and external electric field terms has been considered for ADP crystal. Expressions for shift, width, renormalized antiferroelectric mode frequency, dielectric constant and loss tangent have been evaluated. Double-time thermal Green's function method has been used for derivation. Fitting the values of model parameters in expressions, the temperature dependences of antiferroelectric mode frequency dielectric constant and loss tangent have been calculated in presence of electric field. Theoretical results agree with experimental data by others.
\end{abstract}

Keywords: Antiferroelectrics, Anharmonic interactions, Green Function, Electric field

\section{Introduction}

Ammonium dihydrogen phosphate $\left(\mathrm{NH}_{4} \mathrm{H}_{2} \mathrm{PO}_{4}\right)$ ferroelectric crystals have got large applications. ADP crystal undergoes a first order transition at $148 \mathrm{~K}$, accompanied by tetragonal $\left(\mathrm{D}_{2}-42 \mathrm{~m}\right)$ to orthorhombic $\left(\mathrm{D}_{2}-222\right)$ below $\mathrm{T}_{\mathrm{c}}$. The $\left(\mathrm{H}_{2} \mathrm{PO}_{4}\right)^{-1}$ network in which each phosphate group is linked by $\mathrm{O}-\mathrm{H}$... O bonds to a tetrahedral arrangement of phosphate group neighbours. The lattice parameters in AFE phase $(77 \mathrm{~K})$ are $a=7.507 \AA$, $b=7.529 \AA$ and $\mathrm{c}=7.445 \AA$ and in PE phase $\left(22^{\circ} \mathrm{C}\right)$ are $\mathrm{a}=\mathrm{b}=7.502 \AA \hat{\AA}, \mathrm{c}=7.520 \AA \hat{\AA}$. A large isotope shift in transition temperature from 148 to $242 \mathrm{~K}$ occurs in ADP when it is deuterated. In ADP, below $\mathrm{T}_{\mathrm{c}}$ protons are ordered which produce an antiparallel arrangement of electric dipoles in a- (or b-) axis direction. Ordered ADP has one upper and one lower site for an arbitrary $\mathrm{H}_{2} \mathrm{PO}_{4}$ group filled.

These positions are taken up to produce a perfectly ordered arrangement of bonds in an antiferroelectric pattern. Theoretically Banerjee et al., ${ }_{1}^{1}$ using Green's function method have studied antiferroelectric transition and dielectric properties of ADP-type crystals.

Kim and Sherman ${ }^{2}$ have made Raman studies on mixed ADP crystal. Meena and Mahadevan $^{3}$ have made growth and electrical characterization studies of ADP and $L$-arginine doped ADP crystal. Jayarama et al. ${ }^{4}$ have made neutron diffraction studies of 
thiourea doped ADP crystals. Electric field has been shown to drastically affect the antiferroelectric and dielectric properties of antiferroelectric crystals.

Okada et al., ${ }^{5}$ have carried out experimental studies of effect of applying electric field on dielectric properties of similar antiferroelectric crystal copper formate tetrahydrate. The main aim of the present work is to see the effect of electric field on dielectric constant and loss tangent in ADP crystal.

In the present study a four sublattice pseudospin ${ }^{1}$ lattice coupled mode model ${ }^{1}$ modified along with third and fourth order phonon anharmonic interactions terms and external electric field terms has been used to obtain expressions for shift, width, renormalized antiferroelectric mode frequency, dielectric constant and loss tangent. Okada et al., ${ }^{5}$ have developed thermodynamic Gibbs function theory for ferroelectrics of ADP and copper formate tetrahydrate and calculated susceptibility for d.c. electric field. They have mentioned in their paper that their data agree with experimental data for ADP and CFT. The method of double time thermal Green's function ${ }^{6}$ has been used for the calculation. By fitting model values ${ }^{1}$ of various paramaters appearing in expressions, obtained for ADP crystal. Temperature and electric field dependences of dielectric constant and loss tangent have been calculated. Our findings agree with theoretical data of Okada et al., ${ }^{5}$ which agree with experimental observations for ADP and copper formate crystals.

\section{Theory}

For antiferroelectric ADP crystal, the four-sublattice pseudospin-lattice coupled mode model is modified with third and fourth order phonon anharmonic interaction terms and electric field terms which is expressed as;

$$
\begin{aligned}
& H=-2 \Omega \sum_{i} S_{i}^{x}-2 \Omega \sum_{a}\left(S_{i(1)}^{x(+a)}-S_{i(1)}^{x(-a)}\right)-2 \Omega \sum_{b}\left(S_{i(2)}^{x(+a)}-S_{i(2)}^{x(-a)}\right)-\frac{1}{2} \sum_{i j} J_{i j} S_{i}^{z} S_{j}^{z}-\sum_{i j} \gamma S_{i}^{z} S_{j}^{z}-\mu_{c} E_{c} \sum S_{i}^{z} \\
& -\left(\mu_{s} E_{a}-\frac{1}{2} \lambda\right) \sum_{a}\left\langle S_{(2)}^{z(+a)}-S_{(2)}^{z(-a)}\right\rangle\left(S_{i(1)}^{z(+a)}-S_{i(1)}^{z(-a)}\right)-\left(\mu_{q} E_{a}-\frac{1}{2} \lambda\right) \sum_{a}\left\langle S_{(1)}^{z(+a)}-S_{(1)}^{z(-a)}\right\rangle\left(S_{i(1)}^{z(+a)}-S_{i(1)}^{z(-a)}\right) \\
& -\left(\mu_{z} E_{a}-\frac{1}{2} \lambda\right) \sum_{b}\left\langle S_{(2)}^{z(+b)}-S_{(2)}^{z(-b)}\right\rangle\left(S_{i(1)}^{z(+b)}-S_{i(1)}^{z(-b)}\right)-\left(\mu_{a} E_{a}-\frac{1}{2} \lambda\right) \sum_{b}\left\langle S_{(1)}^{z(+b)}-S_{(1)}^{z(-b)}\right\rangle\left(S_{i(1)}^{z(+b)}-S_{i(1)}^{z(-b)}\right) \\
& -\sum_{i k} V_{i k} S_{i}^{z} A_{k}-\sum_{a} V_{i k}\left(S_{i(1)}^{z(+a)}-S_{i(1)}^{z(-a)}\right) A_{k}-\sum_{b} V_{i k}\left(S_{i(1)}^{z(+b)}-S_{i(1)}^{z(-b)}\right) A_{k} \\
& +\frac{1}{4} \sum_{k} \omega_{k}\left(A_{k} A_{k}^{+}+B_{k} B_{k}^{+}\right)+\sum_{k_{1} V_{2} V_{3}}^{(3)}\left(k_{1}, k_{2}, k_{3}\right) A_{k_{1}} A_{k_{2}} A_{k_{3}}+\sum_{k_{1} k_{2} k_{3} k_{4}} V^{(4)}\left(k_{1}, k_{2}, k_{3}, k_{4}\right) A_{k_{1}} A_{k_{2}} A_{k_{3}} A_{k_{4}}
\end{aligned}
$$

Where $\Omega$ is proton tunnelling frequency, $S^{z}$ and $S^{x}$ are components of pseudospin variable $S, V_{i k}$ is spin-lattice interaction, $A_{k}$ and $B_{k}$ are position and momentum operators, $\omega_{\mathrm{k}}$ is harmonic phonon frequency, $\mathrm{V}^{(3)}$ and $\mathrm{V}^{(4)}$ are third-and fourth-order atomic force constants, $\lambda$ is antiferroelectric interaction constant, $\mathbf{J}$ is exchange interaction, $\gamma$ is long range interaction constant, $\mu$ is dipole moment of $\mathrm{O}-\mathrm{H}-\mathrm{O}$ bond and $\mathrm{E}$ is electric field.

Following Zubarev ${ }^{6}$ we consider the evaluation of Green's function

$$
G_{i j(1)}^{z(+a)}\left(t-t^{\prime}\right)=\left\langle\left\langle S_{i(1)}^{z(+a)} ; S_{j(1)}^{z(+a)}\right\rangle\right\rangle=-i \theta\left(t-t^{\prime}\right)\left\langle\left[S_{i(1)}^{z(+a)}, S_{j(1)}^{z(+a)}\right]\right\rangle,
$$

Differentiating Green's function [Eq. (2)] with respect to times t and t' respectively twice with the help of model Hamiltonian (1), Fourier transforming and putting in the Dyson's equation form, one obtains finally 


$$
\begin{gathered}
G_{i j}^{z(+a)}(\omega+i \in)=\frac{\Omega\left\langle S_{i}^{x}\right\rangle}{\pi\left[\omega^{2}-\hat{\Omega}^{2}-2 i \Omega \Gamma(\omega)\right]} \\
\hat{\Omega}^{2}=\frac{1}{2}\left[\left(\hat{\omega}_{k}^{2}+\tilde{\widetilde{\Omega}}^{2}\right) \pm \sqrt{\left.\left(\hat{\omega}_{k}^{2}-\tilde{\widetilde{\Omega}}^{2}\right)^{2}+8 V_{i k}^{2} b\left\langle S_{i}^{x}\right\rangle \omega_{k}\right]}\right. \\
\epsilon_{a}(\omega)=-8 \pi N \mu_{a}^{2} \Omega\left\langle S_{i}^{x}\right\rangle\left[\omega^{2}-\hat{\Omega}^{2}-2 \Omega i \Gamma_{1}(\omega)\right]^{-1}, \\
\tan \delta_{a}=\frac{\operatorname{Im} \text { aginary } \epsilon}{\operatorname{Re} \text { al } \epsilon}=-2 \Omega \Gamma_{1}(\omega)\left(\omega^{2}-\hat{\Omega}^{2}\right)^{-1},
\end{gathered}
$$

\section{Numerical calculation and results}

By using model values of various quantities for ADP-crystal given by Banerjee et al ${ }^{l}$., $\mathrm{T}_{\mathrm{c}}=$ 148K, $\Omega=45 \mathrm{~cm}^{-1}, \lambda=90 \mathrm{~cm}^{-1},(\mathrm{~J}+\gamma)=180 \mathrm{~cm}^{-1}, V_{\mathrm{ik}}=40 \mathrm{~cm}^{-1}, \mu \times 10^{18}=4.70 \mathrm{cgs}$, $\mu \times 10^{18}=2.95 \mathrm{cgs}, \omega_{\mathrm{k}}=153 \mathrm{~cm}^{-1}$, the temperature and field dependences of dielectric constant and loss tangent have been calculated and shown in Figure 1-2. The theoretical results agree with experimentally reported findings of Okada $e t a l^{5}$. Who studied effect of electric field on dielectric constant in ADP and copper formate crystals. The effect of electric field on antiferroelectric mode frequency \& loss tangent of ADP crystal has been obtained by correlating experimental findings of Okada et al., ${ }^{5}$ for dielectric constant of ADP crystal. It can be seen from our theoretical data that the dielectric constant $\left(\varepsilon_{\mathrm{a}}\right)$ decrease with increase in electric field strength (in paraelectric phase) [see eq 5] and the tangent losses $\left(\tan \delta_{\mathrm{a}}\right.$ ) decrease with increase in electric field strength [see eq 6]. These observations are in good agreement with experimental findings of Okada et $a l^{5}$.

Table 1. Calculated values of $\mu E$ for ADP crystal (Banerjee et al. ${ }^{1}$ )

\begin{tabular}{ccccccccccc}
\hline $\mathrm{E}(\mathrm{kV} / \mathrm{cm})$. & 1 & 2 & 3 & 4 & 5 & 6 & 7 & 8 & 9 & 10 \\
\hline $\begin{array}{c}\mu_{\mathrm{a}} \mathrm{E}_{\mathrm{a}} \\
\left(\mathrm{kJ} / \mathrm{cm}^{3}\right)\end{array}$ & 0.088 & 0.175 & 0.262 & 0.350 & 0.437 & 0.525 & 0.612 & 0.700 & 0.787 & 0.875 \\
\hline
\end{tabular}

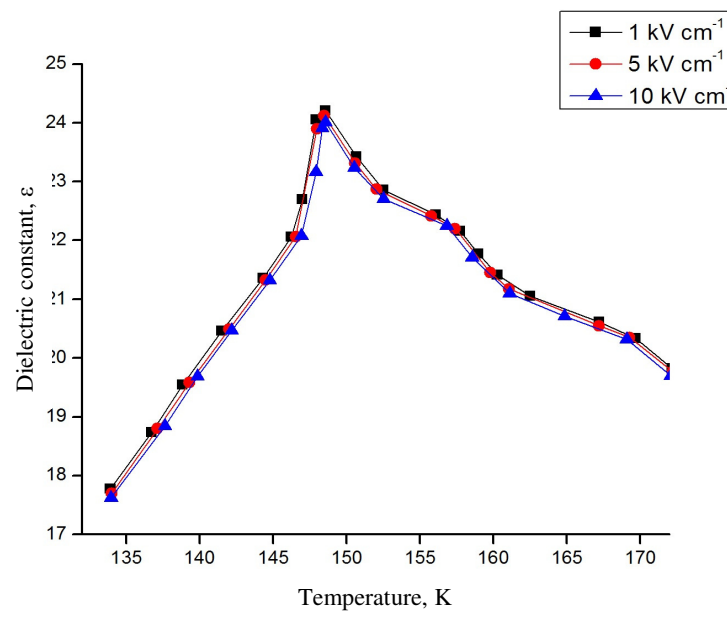

Figure 1. Temperature dependence of dielectric constant in ADP crystal, present calculation-, Okada et al's data ${ }^{5}$ 


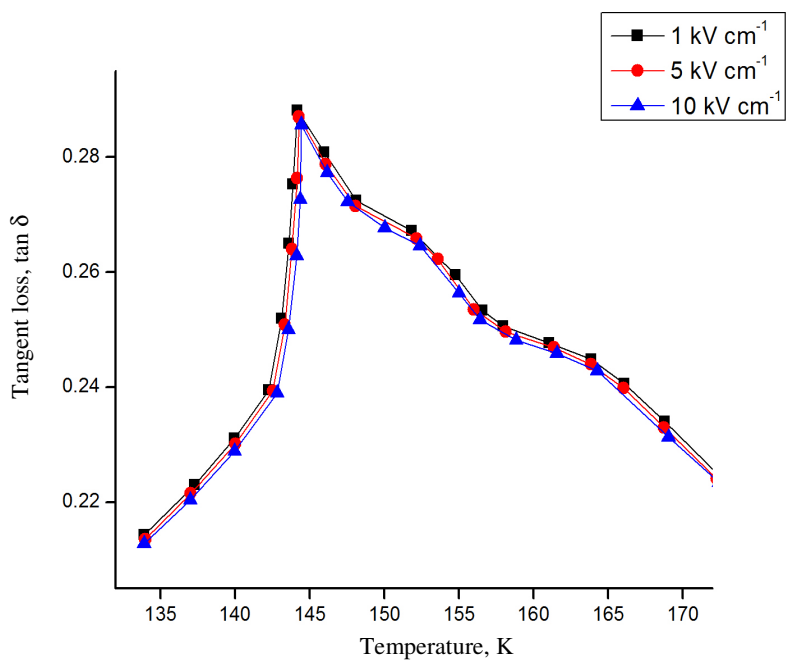

Figure 2. Temperature dependence of dielectric loss in ADP crystal, present calculation-, Okada et al's data ${ }^{5}$

\section{Results and Discussion}

In the present work, by considering four sublattice pseudospin lattice coupled mode model, along with third- and fourth- order phonon anharmonic interaction terms, expressions have been derived theoretically for dielectric constant and loss tangent for ADP-type antiferroelectric crystals. Method of double-time thermal Green's function has been used for the derivation of response function. Earlier researchers have not considered the third order phonon anharmonic interaction term in their model Hamiltonian and they have not discussed effect of electric field on antiferroelectric and dielectric properties. They decoupled the correlations at an early stage, due to which some important interactions disappeared from their expressions. Our data agree with data of Okada et $a l^{8-10}$. The physical explanation to this effect is that in presence of electric field (d.c. bias) crystal behaves as clamped crystal. The d.c. field affects the force constant or positions of ions. This in some way decreases the time period of oscillations of ions or increases their normal mode frequencies.

\section{Conclusion}

Our data show that both dielectric constant and loss tangent decrease with increase in electric field strengths. This agrees with data of Okada et $a l^{5}$. The physical explanation of this is that the electric field does the exclusion of domains in crystal. As a result this decreases dielectric constant and loss of the crystal. It emerges from the present study that the effect of electric field is to reduce the values of dielectric constant and loss tangent at each temperature.

\section{Acknowledgement}

Authors are grateful to Eminent Physicist Prof. B S Semwal(Former HOD Physics) for his blessings and to Prof. R P Gairola(Former HOD Physics), Prof. U C Naithani(HOD Pauri Campus), Prof. S C Bhatt(HOD Physics) for their encouragements. One of the author (DJ) is thankful to his parents for their support and to Mr Prabhat Khanduri, Miss Aanchal Rawat, Mrs Deepali Raturi, Mrs Anubhuti Mamgain for their encouragements. 


\section{References}

1. Banerjee S, Nath D and Chaudhuri B K, Phys Rev B, 1981, 24, 6469; DOI:10.1103/PhysRevB.24.6469

2. Kim J J and Sherman W F, Phys Rev B, 1987, 36, 5651; DOI:10.1103/PhysRevB.36.5651

3. Meena M and Mahadevan C K, Cryst Res Technol., 2008, 43(2), 166-172; DOI:10.1002/crat.200711064

4. Jayarama A, Suresh Kumar M R, Dharmaprakash S M, Chitra R and Choudhury R R, Pramana J Physics, 2008, 71(5), 905-910; DOI:10.1007/s12043-008-0198-6

5. Okada K, Sugie E, Kanno K and Tada K, J Phys Soc Jpn., 1977, 43, 557-562; DOI:10.1143/JPSJ.43.557

6. Zubarev D N, Sov Phys Usp., 1960, 3(3), 320; DOI:10.1070/PU1960v003n03ABEH003275

7. Kubo R, J Phys Soc Jpn., 1957, 12, 570-586; DOI:10.1143/JPSJ.12.570

8. Okada K, J Phys Soc Jpn, 1969, 27, 420-428; DOI:10.1143/JPSJ.27.420

9. Okada K, J Phys Soc Jpn, 1974, 37, 1226-1232; DOI:10.1143/JPSJ.37.1226

10. Okada K and Sugie H, J Phys Soc Jpn, 1968, 25, 1128-1132; DOI:10.1143/JPSJ.25.1128 